\title{
Cell search and synchronization in 5G NR
}

\author{
Yakov Kryukov ${ }^{1, *}$, Dmitriy Pokamestov ${ }^{1}$, and Eugeniy Rogozhnikov ${ }^{1}$ \\ ${ }^{1}$ Tomsk State University of Control Systems and Radioelectronics, Department of \\ Telecommunications and Basic Principles of Radio Engineering, 634050 Tomsk, Russia
}

\begin{abstract}
An overview of the physical signals foreseen by the 3GPP 5G New Radio specification for frame synchronization and cell search in fifthgeneration wireless broadband access systems is presented in the paper. The frame synchronization algorithm and the cell initialization procedure are demonstrated. An estimate of probability of error detection of a physical identifier by the signals of the primary and secondary synchronization is obtained. The comparison of the successful synchronization in AWGN channel for 4G LTE and 5G NR is shown.
\end{abstract}

\section{Introduction}

In September 2018, the 3GPP published the 15th release of the technical specification ETSI 38.211 [1], which describes the physical layer procedures of 5G New Radio (NR) communication systems [2]. One of the requirements of 5G NR is providing the ability for quick radioframe synchronization to reduce the user equipment connection time to the base station network. To achieve this, developers have to increase the complexity and reduce the transmit periodicity of synchronization signaling. Therefore, for NR is allowed using from 8 to 64 synchronization blocks during the radioframe. Using any detected block the user equipment (UE) synchronize and immediately transmit an access request to the base station (eNB). In this regard, the synchronization procedure is significantly changed compared to $4 \mathrm{G}$ LTE [3], and for synchronization not the two simple signals are used, but the signal block which consist of few signals.

The paper describes the synchronization signals generation method and discusses cell search and radioframe synchronization procedures. The estimate of the noise immunity of the synchronization signals is obtained from the result of mathematical simulation and it is shown in the experimental part. The article shows the calculation result of the value of the signal-to-noise ratio at the input of the correlation receiver at which synchronization procedure can be successfully done with the required error probability.

\section{Sychronization signal block}

Synchronization Signal Block (SSB) [1] consists of three special signals and one physical channel. The one SS block is occupies 300 subcarriers in each of 4 OFDM symbols in the

* Corresponding author: kryukov.tusur@gmail.com 
time-frequency domain. In other words, 1200 resource elements (RE) of the resource grid are used to transfer the one synchronization block (versus $288 \mathrm{RE}$ for LTE). The structure of the synchronization block with the subcarrier indices and the OFDM symbol numbers of each SSB part is presented in fig. 1.

This block structure provides the possibility of frame synchronization by using the detected block location number within radioframe from each received SSB. The first three bits (iSSB ${ }_{1}$ field) are transmitted by the demodulated reference signal, and the second three bits (iSSB ${ }_{2}$ field) are transmitted in the physical broadcast channel. The following is a brief overview of signals in synchronization block.

The type of synchronization signal is individual for each cell (as LTE) and depends on unique physical-layer cell identities value $N_{I D}^{\text {cell }}=3 \cdot N_{I D}^{1}+N_{I D}^{2}$ in the range of 0 to 1008 . The physical-layer cell identities are grouped into 336 unique physical-layer cell-identify groups $\left(\mathrm{N}_{\text {ID }}{ }^{1}\right.$ value), and each group containing three unique identities $\left(\mathrm{N}_{\mathrm{ID}}{ }^{2}\right.$ value). The grouping is such that each physical-layer cell identity is part of one and only one physical-layer cellidentity group.

Primary Synchronization Signal (PSS) is a kind of binary pseudo-random m-sequence with a duration of 127 samples, which is formed depending on the number $\mathrm{N}_{\text {ID }}{ }^{2}$ in the range of 0 to 2, representing the physical-layer identity within the cell-identity group. PSS is always located in the first OFDM symbol of synchronization block and occupies subcarriers with indexes from 57 to 183.

Secondary Synchronization Signal (SSS) with a duration of 127 samples is generated from combination of two m-sequences, which are generated depending on the group identifier $\mathrm{N}_{\mathrm{ID}}{ }^{1}$ specified in the range of 0 to 335. SSS is always located in the third OFDM symbol of synchronization block (like PSS) and occupies subcarriers with indexes from 57 to 183 too.

Demodulated Reference Signal (DMRS) is a QPSK modulated m-sequence based on sequence number iSSB $_{1}$ of the SSB location in a radioframe. DMRS symbols are located on every 4th subcarrier in each synchronization block OFDM symbol. DMSS occupies 144 REs within the synchronization block.

Physical Broadcast Channel (PBCH) transmits 4 common information fields with service data, which must be demodulated by each user. The 56 information bits are transmitted by $\mathrm{PBCH}$ in each SSB, of which: the last 24 bits are the cyclic redundancy check (CRC) and the 24 first bits are used for detection of the primary parameters of the cell configuration. Using the remaining bits, the UE can find sequence number of the SSB in the frame, after which it becomes possible to detect of the radioframe beginning and then to start procedure of time synchronization. QPSK modulation and polar coding [4] with high redundancy are used in the $\mathrm{PBCH}$ to improve noise immunity. Therefore, $\mathrm{PBCH}$ has a high noise resistance, and 432 REs are used to transmit a 32-bit message. Unused RE from each side are the guard interval in frequency domain.

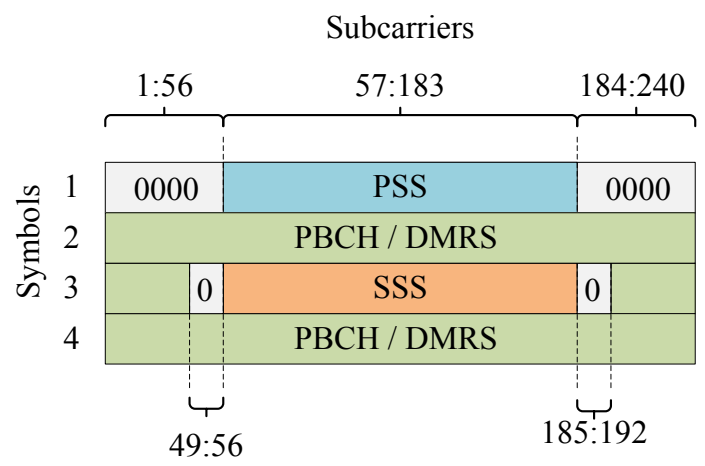

Fig. 1. Structure of SSB. 


\section{Radioframe synchronization and cell search}

The block diagram of the frame synchronization procedure is shown in fig. 2 . The first step of the synchronization procedure at the UE side is the detection PSS and the determination of the $\mathrm{N}_{\mathrm{ID}}{ }^{2}$ value. Thereafter, a bank of 336 secondary synchronization signals is generated on the base of detected $\mathrm{N}_{\mathrm{ID}}{ }^{2}$ then detection of SSS in received signal and determination of the $\mathrm{N}_{\mathrm{ID}}{ }^{1}$ value is performed. After both of $\mathrm{N}_{\mathrm{ID}}{ }^{1}$ and $\mathrm{N}_{\mathrm{ID}}{ }^{2}$ are detected, the cell ID is calculated and the location of the $\mathrm{PBCH}$ in the resource (time-frequency) grid is determined. A new cell with the Cell ID physical identity and the received signal power estimation is registered in a table of the UE available cells. The new base station tracking begins at this stage.

A DMRS signal bank is generated based on the Cell ID value. The UE determines the first part of the iSSB bits (iSSB ${ }_{1}$ field) by the DMRS correlation detecting and estimates the channel coefficients for $\mathrm{PBCH}$ equalization with further $\mathrm{PBCH}$ demodulation. As a result

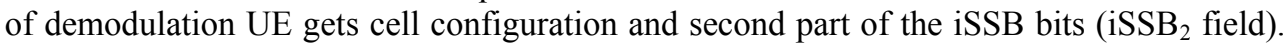
The SSB sequence number (iSSB value) in the frame is calculated based on both of iSSB $_{1}$ and $\mathrm{iSSB}_{2}$. It becomes possible to calculate the frame time interval and realize frame synchronization.

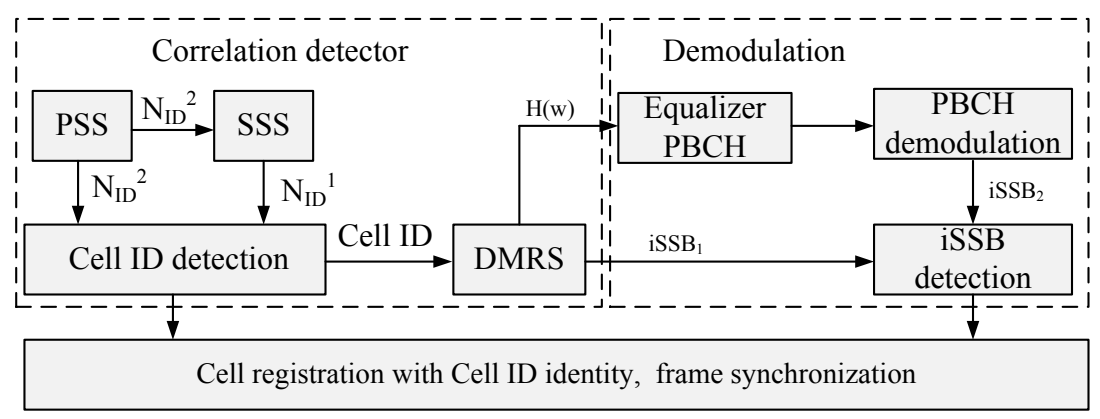

Fig. 2. Radioframe sycnrhonization procedure.

\section{Analysis of synchronization signals noise resistance}

The transmission of synchronization signals is based on the direct spread spectrum technology [5], which provides additional system gain by correlation processing at the receiver side. The PSS and SSS has a spreading factor of 127 (system gain $21.03 \mathrm{~dB}$ ), and the DMRS has value of 144 (system gain $21.58 \mathrm{~dB}$ ). System gain allows to detect these signals even at low power and below the noise floor.

The purpose of the mathematical simulation was to obtain the probability of false detection of the parameters of each signal in the synchronization block at the UE side, provided that the parameters of the other signals of the block were are correctly detected. Within the mathematical simulation the generation, demodulation and transmission of the SSB via the AWGN channel, which are carried out according to the method described in Section 3, are occurred.

Successful frame synchronization is achieved with successful demodulation of $\mathrm{PBCH}$. The dependence of the error demodulation probability of signal parameters $\left(\mathrm{N}_{\mathrm{ID}}{ }^{2}, \mathrm{~N}_{\mathrm{ID}}{ }^{1}\right.$, id1, id2) from the signal-to-interference-and-noise ratio in the AWGN channel is shown in Fig. 3. The simulation result showed that frame synchronization with an error probability of less than $10^{-3}$ is achieved with a signal-to-interference-and-noise ratio (SINR) value at the input of the correlation receiver of at least $-5 \mathrm{~dB}$ (via PBCH). Cell detection with a false detection probability of less than $10^{-3}$ is achieved with SINR value at the input of the correlation receiver of at least $-7.35 \mathrm{~dB}$ (refer to SSS). For comparison, the same probability of cell 
detection and frame synchronization is achieved with an SINR of at least $-4.35 \mathrm{~dB}$ in the 4G LTE system.

Analyzing the simulation result, it is possible to conclude that successful 5G NR frame synchronization requires the SINR value in the channel $0.65 \mathrm{~dB}$ more, and for cell identification $3 \mathrm{~dB}$ less than in the LTE system.

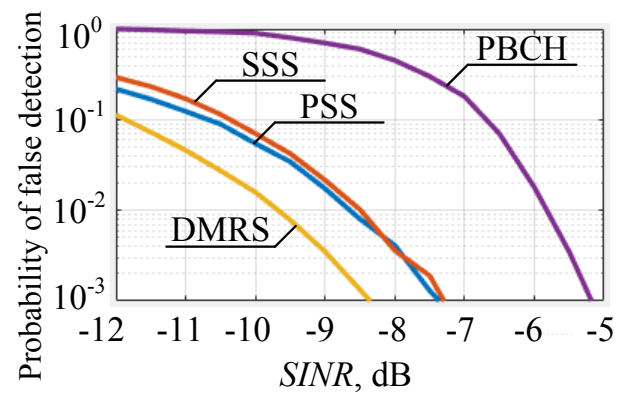

Fig. 3. False detection probability of SBB parameters.

\section{Conclusion}

The paper presents an overview of the frame synchronization and $5 \mathrm{G}$ NR cell search procedure by signals, which are provided for by the ETSI 38.211 (Release 15) technical specification. In comparison with LTE for frame synchronization in NR it is necessary to research complex processing of the synchronization block consisting of 3 signals and the 1 channel (versus 2 simple signals in LTE).

The simulation result showed that for synchronization in the NR at the input of the correlation receiver, the SINR value is required by $0.65 \mathrm{~dB}$ less than in LTE. However, for LTE transmission synchronization signal resource elements are allocated less than NR. Due to the increase of the synchronization signals duration (compared to LTE), the accuracy of estimating time and frequency synchronization errors is increased too. Also, the UE earlier begins the procedure of getting access to the base station network due to the more frequent transmission of the synchronization block in radioframe.

The work is supported by the Russian Federation President grant to ensure young Russian scientists research state support. Grant number MK-1126.2019.9.

\section{References}

1. 3GPP TS 38.211 NR; Physical channels and modulation (2018)

2. S. Y. Lien., S.L. Shieh, Y. Huang, B. Su, Y.L. Hsu, H.Y. Wei $5 G$ new radio: Waveform, frame structure, multiple access, and initial access, IEEE communications magazine, v. 6, pp. 64-71 (2017)

3. E. Dahlman, S. Parkvall, J. Skold 4G: LTE/LTE-advanced for mobile broadband, Academic press (2013)

4. P. Trifonov Efficient design and decoding of polar codes, IEEE Transactions on Communications, v. 11, pp. 3221-3227 (2012)

5. R. C. Dixon Spread spectrum systems: with commercial applications (WileyInterscience, New-York, 1994)

6. X. Yang, Y. Xiong, G. Jia, W. Fang, X. Zheng PSS based time synchronization for $3 G P P$ LTE downlink receivers, 2011 IEEE 13th International Conference on Communication Technology, pp. 930-933 (2011) 